\title{
DESIGN OF NEW SELF-RESETTING NEGATIVE STIFFNESS MECHANISM FOR SHOCK ABSORPTION BASED ON FINITE ELEMENT METHOD
}

\author{
Huijuan $\mathrm{Li}^{1}$ \\ ${ }^{1}$ Zhengzhou Vocational College of Finance and Taxation, Henan 450000, China \\ Email: lihuijuan198@163.com
}

\begin{abstract}
In order to further improve the seismic performance of the existing negative stiffness mechanism, an improved self-resetting negative stiffness mechanism with auxiliary energy dissipation elements parallel to the pre-pressure spring is developed. By summarizing the research status of self-reset energy dissipation brace, the basic structure and working principle of the brace are described. Based on the quasi-static test results of the brace specimens, the finite element modelling and numerical analysis of hysteretic performance of self-reset energy dissipation brace are carried out, and the relevant design parameters of the brace are analyzed. At the same time, focusing on the actual seismic reduction and reinforcement projects, the finite element simulation method is used to conduct a comprehensive study on the analysis method and seismic effect of energy dissipation structures with self-resetting energy dissipation braces. The results show that the stiffness of preloading spring not only affects the development process of negative stiffness on structural weakening and vibration reduction, but also affects the effect of strengthening self-reset of structure after decompression. The design value of preloading displacement only affects the development process of structural weakening and seismic reduction with negative stiffness. The auxiliary energy dissipation elements parallel to the pre-loaded spring can provide the ability to dissipate seismic energy. In this way, the seismic absorption effect of the structure can be further enhanced. Therefore, the improved self-resetting negative stiffness mechanism achieves the performance design goal of organic unification of shock absorption, energy dissipation capacity and self-resetting effect.
\end{abstract}

Keywords: Seismic Self-Reset; Finite Element Analysis; Seismic Performance; Effective Shock Absorption.

\section{Introduction}

According to statistics, more than 5 million earthquakes occur on the earth every year, which means tens of thousands of earthquakes occur every day. Many of these earthquakes are too small or the focal depth is too deep to be noticed. There are about ten or twenty earthquakes that can really cause serious harm to human beings every year. Great earthquakes often cause serious casualties and huge economic losses to human beings and society. Although the disasters caused by earthquakes cannot be completely avoided, building structures designed for earthquake resistance can achieve the goal of not falling down in large earthquakes. Therefore, controlling the damage degree of the structure under earthquake, improving the seismic performance of the structure, and putting forward more advanced seismic design methods has been the focus of research in the field of earthquake resistance.
In the past, structural seismic resistance was a passive and negative mean of seismic resistance. It was the structure itself that stored and consumed seismic energy. In view of the shortcomings of traditional seismic design methods, most of them are dissipated energy of the structure itself, which may cause serious safety problems to the structure [1]. The ideal building structure should have the ability to recover quickly after the exhibition, so that people's life after the earthquake will not be affected. At present, the maximum deformation is usually used as the index to measure the seismic performance of building structures, and the control of maximum deformation is the basic goal of seismic design. However, many building structures cannot meet the requirement of continued use because of large residual deformation after exhibition. The building structures with excessive residual deformation cannot be repaired to normal use, and can only be demolished eventually. Therefore, reducing or even eliminating the residual 
deformation of building structure after earthquake is also an important goal of structural seismic design.

\section{Literature Review}

Energy dissipation technology has been successfully applied to the seismic control of engineering structures because of its clear mechanism, remarkable effect, safety and reliability, reasonable economy and wide application range [2] .

In 2015, Singh and Satpute pointed out that energy dissipation and seismic reduction technology based on traditional design can greatly improve the seismic, wind and seismic capacity of buildings and structures, effectively reduce the response and damage of wind and earthquake to structures, and improve the seismic capacity and seismic performance of structures [3]. In 2016, Li et al. proposed a method for seismic strengthening of four-to six-storey frame structures with friction dampers, which are located in the 8-degree earthquake intensity zone and site II [4]. In 2015, Pu et al. used friction dampers to analyze and calculate the seismic strengthening of frame structures with six stories, which effectively improved the seismic performance of structures.

This method will help structural design engineers and researchers [5]. In 2015, Travanca and Hong studied the dynamic deformation and failure process of slopes with weak interlayer under earthquake by shaking table model test. The test results show that the deformation and failure process of slopes under earthquake is related not only to the type of seismic wave, load direction and vibration intensity, but also to the elevation of slopes and the thickness of weak interlayer [6]. In 2016, Correagalvis et al. designed a special shock absorber based on vibration isolation technology, which greatly improved the isolation effect by connecting the isolation bearing and elastic steel ball [7]. In 2015, in order to reduce the seismic response of structures, Martnez-Hergueta et al. developed a passive energy dissipation device, velocity and displacement related hydraulic damper (VDHD) [8]. In 2017, Luna et al. carried out various acceleration vibration analysis of reinforced structures, and analyzed their dynamic characteristics and vibration reduction effect [9].

In 2017, Bashtovoi et al. combined with the seismic response characteristics, proposed the method of setting fluid viscous dampers at the joints between columns and roof, and analyzed the time history of a reservoir with fluid viscoelasticity [10].

In 2018, Mohammadzadeh-Habili et al. made a preliminary study on a composite buckling structure of elastic hysteretic elements with high energy absorption efficiency and energy dissipation efficiency. In order to clarify its basic idea, a conceptual model composed of two-bar linkage and hysteretic spring was studied [11].

\section{Methodology}

Active seismic design method is to install shock absorption and isolation devices on the structure.

The structures and devices bear seismic load together and absorb and dissipate seismic energy. Active seismic design theory is the most effective and reasonable way to improve the seismic performance of structures. There are many methods for vibration control of engineering structures, which can be roughly divided into passive control, active control, semi-active control and hybrid control according to whether there is external energy input or not, as shown in figure 1.

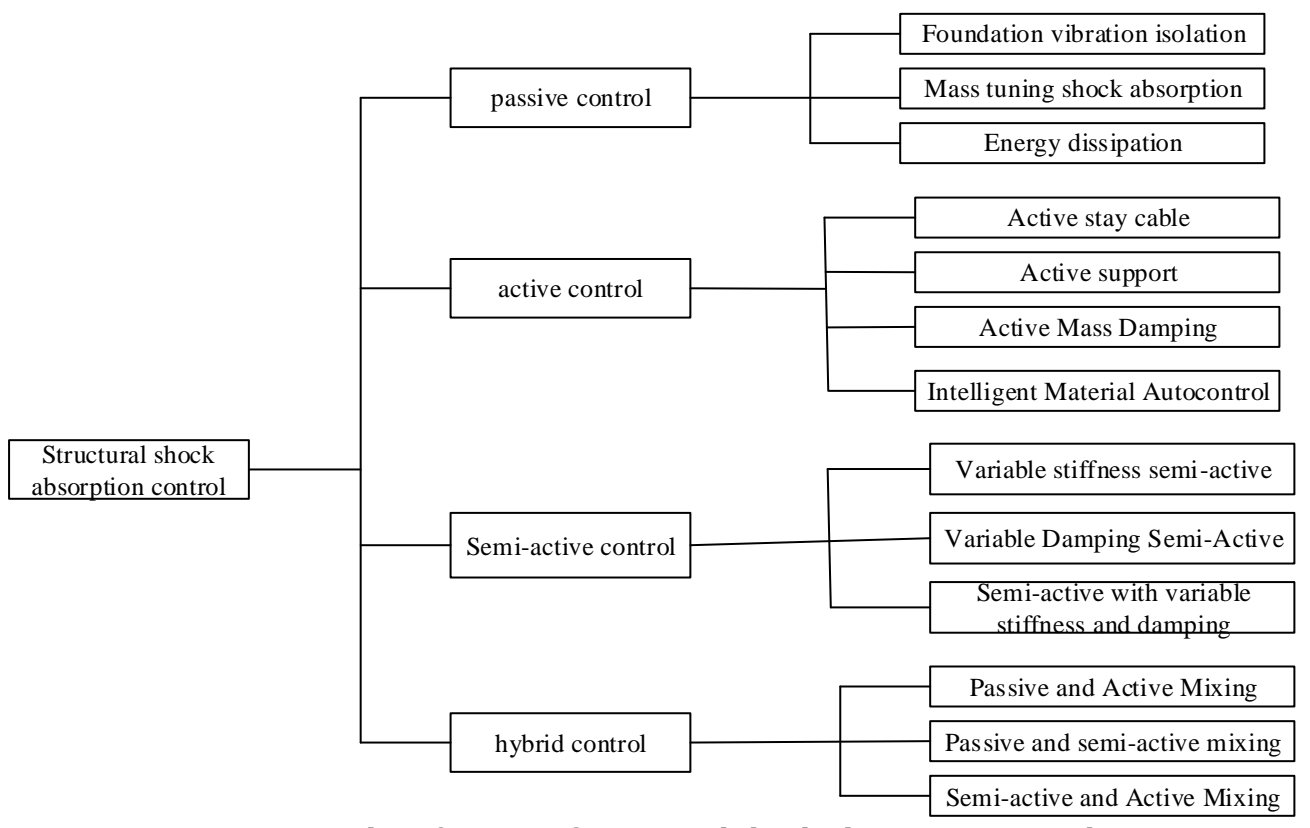

Figure 1: Classification of structural shock absorption control 
The working mechanism of self-resetting energy dissipation support under tension and compression is mainly realized by the interaction between inner core, outer cylinder, end plate, aramid fiber bundle and friction energy dissipation device. The aramid fiber bundles are always stretched regardless of the tension or compression state of the self-resetting energy dissipation support.

The assembly method of this component can make the reset device always provide restoring force for the support, and ingeniously realize the self-reset of the support.

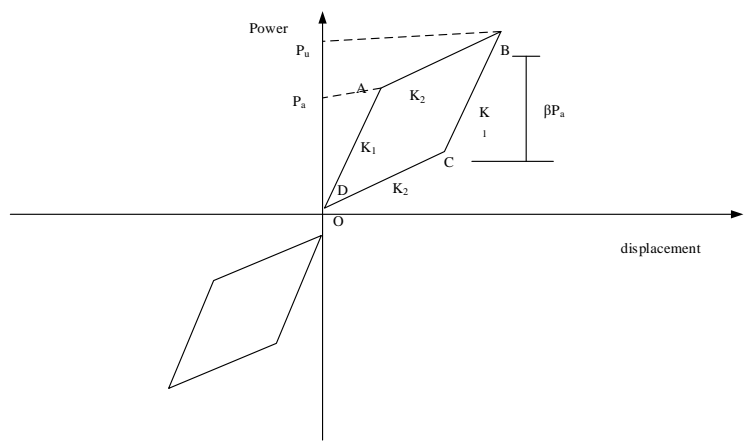

Figure 2: Schematic diagrams of hysteretic curve of self-reset energy dissipation support

Since the hysteretic curve of self-reset energy dissipation support is shaped like two flags, it is called "flag hysteresis". The hysteresis curve in figure 2 is in the tension state of self-resetting energy dissipation support in the first quadrant, and in the compression state, it is in the third quadrant.

The self-reset energy dissipation support consists of reset device, restraint connection device and energy dissipation device. The reset device can eliminate the residual displacement of the support and achieve self-reset effect by applying pre-tension to aramid fiber bundles. The main components of the restraint connection device are inner core, outer tube and end plate, which can transfer the axial force and connect the supporting components. The energy dissipation device uses friction and energy dissipation mechanism, which can dissipate energy when the relative displacement occurs at both ends of the support and play the role of energy dissipation and shock reduction.

\section{Results And Discussion}

\subsection{Basic mechanics principle of negative stiffness damping mechanism}

Taking the ideal linear single-degree-of-freedom structure as an example, its structure is undamped and its stiffness is $K_{\mathrm{e}}$. The stiffness of built-in NSD is $\mathrm{K}_{\mathrm{NSD}}$ and the damping coefficient of passive damper is C. The sketches of AS and NS components are shown in figure 3 , and the stress and displacement curves are shown in figure 4.

For the structural combination with built-in NSD in 4(b), when the structural displacement exceeds $\triangle^{\prime} y$, the stiffness of the structural combination decreases to $\mathrm{K}_{\mathrm{a}}=\mathrm{K}_{\mathrm{e}}-\mathrm{K}_{\mathrm{NSD}}$. The maximum restoring force and displacement of the structure combination corresponding to the maximum restoring force $F_{2}$ and displacement $\triangle 2$ of the ideal linear main structure are transformed into $F_{3}$ and $\triangle 3$, which indicates that adding NSD can effectively achieve the goal of reducing the base shear force, but compared with the elastic system, the maximum deformation of the adaptive system increases obviously in the course of operation.

For the structure combination with NSD and passive damper in figure $3(\mathrm{C})$, NSD can effectively reduce the base shear force, and the maximum displacement of the main structure can be reduced to $\triangle 3$ by using linear viscous damper. When the main structure system and NSD are in the state of elastic force, even if the viscous damper with small damping coefficient is used, the effect is obvious, and the increase of damper is also obvious. At the same time, the addition of dampers has little effect on the base shear of the main structure and the combination of the built-in NSD structure.

From figure 3, it can be seen that the key of setting NSD is the value of the conditional yield displacement $\triangle^{\prime} y$. Reasonable value can avoid excessive response of the structure under small external excitation. When the displacement $|\triangle|<\triangle^{\prime} y$, the NSD does not play its role, and the structure combination shows the main performance of the structure.
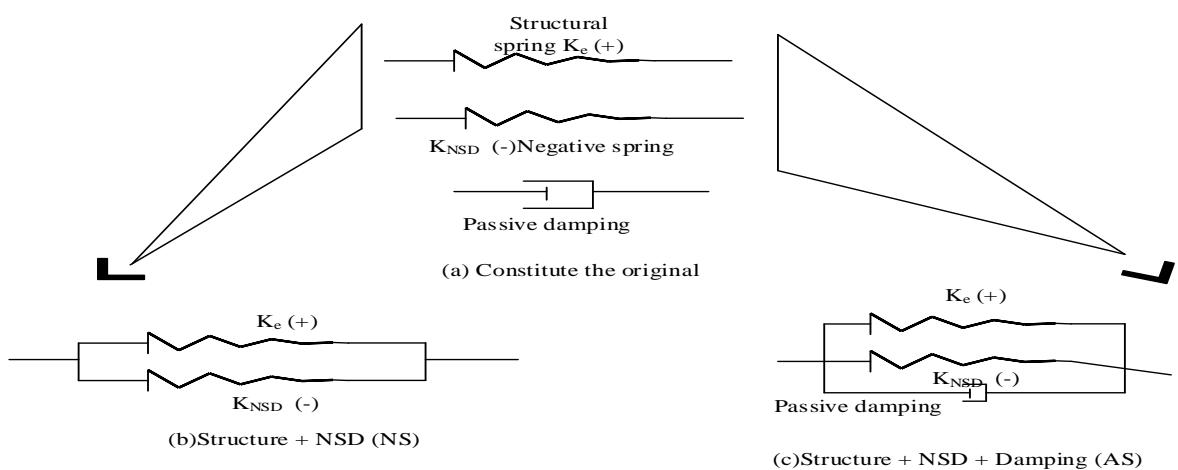

Figure 3: A schematic diagram of AS and NS components 

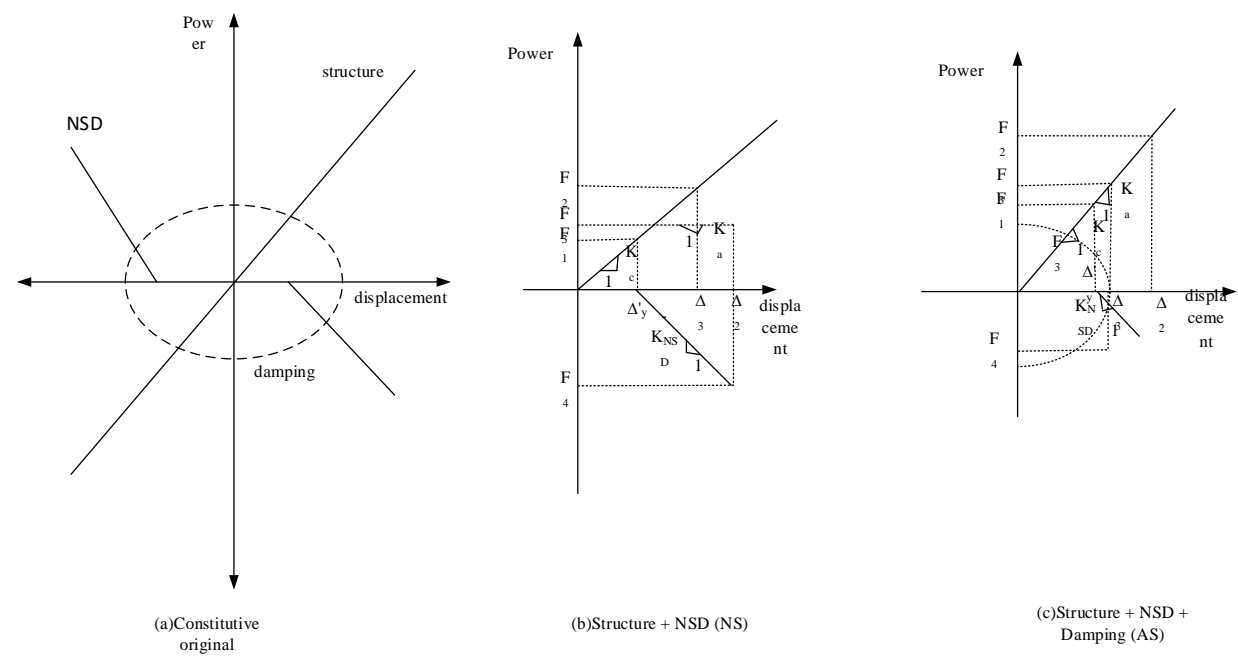

Figure 4: Schematic force-deformation of AS and NS

\subsection{Finite element analysis of modified seismic reduction self-resetting negative stiffness mechanism}

In this section, the finite element model of MNSD is established by using ABAQUS/CAE pre-processing module. Firstly, the components of the improved negative stiffness mechanism (upper herringbone bracket, lower herringbone bracket, double hinged column, rotating pivot plate, arm bar, preload spring and clearance spring and auxiliary energy dissipation element) are modelled separately. Then, rigid body constraints are established between each component and reference point, and then internal connections are made. Connectors defined as hinges are used for connection between rotating pivot plate and lower herringbone bracket, between double hinged column and upper and lower herringbone bracket, and between rotating pivot plate and arm bar. The connection between the lower herringbone bracket and the connection between the rotating pivot plate and the arm bar are all assembled as a whole by using the connector defined as hinge, while the lower herringbone bracket and the foundation are defined as embedded. The hinge connector releases only the translational and rotational degrees of freedom in the plane, while the embedded part restricts all translational and rotational degrees of freedom in the space. The rotating pivot plate, arm bar, upper herringbone bracket, and foundation in the model are assembled as a whole. The solid element C3D8R is adopted for the lower herringbone bracket and double hinged column. The equivalent spring connection element is used for the preloading spring and the clearance spring. The auxiliary energy dissipation element is simulated by the truss element (TRUSS).

The constitutive relationship of materials accurately reflects the main mechanical properties of materials. Therefore, selecting reasonable constitutive relationship of materials is the key to accurate finite element simulation results of structures. In ABAQUS software, there are three steps to assign cross-sectional attributes to components. The first step is to create material attributes, the second step is to create crosssectional attributes and the third step is to assign cross-sectional attributes to components. In this section, the upper herringbone bracket, lower herringbone bracket, double-hinged column, pivot plate and arm bar are high strength steel to ensure that they are in elastic state during the whole process of loading. For this reason, linear elastic model is selected. For yield energy dissipation components, von Mises yield criterion, isotropic strengthening and related flow rule are used.

The cyclic loading is simulated.

$\sigma=\left\{\begin{array}{l}\mathrm{E}_{\mathrm{s}} \varepsilon\left(\varepsilon \leq \varepsilon_{\mathrm{y}}\right) \\ \sigma_{\mathrm{y}}+\mathrm{E}_{\mathrm{st}}\left(\varepsilon-\varepsilon_{\mathrm{y}}\right)\left(\varepsilon>\varepsilon_{\mathrm{y}}\right)\end{array}\right.$

In the formula, $\varepsilon_{y}$ is the yield strain of steel, $\varepsilon_{y}$ is yield stress of steel. Nominal yield strength of steel for energy dissipation element is $100 \mathrm{Mpa}$. $\mathrm{E}_{\mathrm{s}}$ is elastic modulus of steel and its value is $2.06^{*} 10^{5} \mathrm{Mpa}$. $\mathrm{E}_{\text {st }}$ is the modulus of material in strengthening stage.

\subsection{Analysis method of seismic reduction and reinforcement of structures with self- resetting energy-dissipating braces}

China is a country with frequent earthquake disasters. In recent years, there have been several major earthquakes with serious disasters. Many existing buildings have been unable to meet the safety requirements of current codes because of their long disrepair. In this context, the seismic strengthening technology of building structures has become a hot spot of seismic research. As a new seismic strengthening design method, energy dissipation devices are installed to dissipate seismic 
energy, because they can effectively reduce the demolition and reconstruction of building structures after large earthquakes. In addition, these devices have gradually been widely concerned and applied.

A building is taken as a structure with selfresetting energy dissipation braces for seismic reduction and reinforcement. A building is a sixstory frame structure, in which the first to fourth floors are reinforced concrete structures, the fifth and sixth floors are steel structures. There are semibasement floors. The layout of the plane structure of the building is L-shaped, with 8 frames in both horizontal and vertical directions.

By modal analysis of the model structure, the basic performance parameters of the structure can be obtained, which can help us qualitatively judge the response of the structure and provide a reference for the conceptual design of the structure. In addition, modal analysis can also provide relevant structural performance parameters for structural static analysis, such as static seismic analysis, static wind load analysis and so on. Modal analysis is also the basis of structural dynamic analysis, such as response spectrum analysis, time history analysis and so on.

Table 1. Periods of the first 10 modes of the model

\begin{tabular}{|c|c|c|}
\hline $\begin{array}{c}\text { Mode of } \\
\text { vibration }\end{array}$ & $\begin{array}{c}\text { Cycle } \\
(\mathrm{Sec})\end{array}$ & Mode description \\
\hline 1 & 1.309825 & X-Direction translation \\
\hline 2 & 1.265008 & Y-direction translation \\
\hline 3 & 1.071156 & Torsion around Z-axis \\
\hline 4 & 0.505599 & X-Direction translation \\
\hline 5 & 0.474979 & Y-direction translation \\
\hline 6 & 0.404857 & Torsion around Z-axis \\
\hline 7 & 0.321701 & X-Direction translation \\
\hline 8 & 0.303264 & Y-direction translation \\
\hline 9 & 0.266924 & Torsion around Z-axis \\
\hline 10 & 0.246369 & X-Direction translation \\
\hline
\end{tabular}

However, there is no available restoring force model suitable for self-reset energy dissipation support in the unit library of SPA2000, a general finite element analysis software. The simulation of self-reset energy dissipation support is realized by using linear connection element, multi-segment linear elastic connection element and multi-segment linear plastic connection element in SPA2000 connection element. Specific simulation methods are as follows. A multi-segment linear elastic connection element and a multi-segment linear plastic connection element are paralleled, and connected in series with a linear connection element. Then, the flag hysteretic performance of self-reset energy dissipation support is simulated by defining the parameters of the three connection elements.

\subsection{Time history analysis of structures strengthened by shock absorption}

The design of energy dissipation structures should meet the requirements of anticipated vibration reduction under frequent earthquakes and the control requirements of anticipated displacement under rare earthquakes, which are closely related to the calculation and analysis of energy dissipation structures. Time history analysis method, as the main calculation and analysis method of energy dissipation and shock absorption structures stipulated in Code for Seismic Design of Buildings, is suitable for linear analysis under frequent earthquakes and non-linear analysis under rare earthquakes. Structural response analysis under frequent earthquakes uses inter-story displacement angle, absolute acceleration of floor and bottom shear as analysis indexes, while structural response analysis under rare earthquakes uses inter-story displacement angle, inter-story residual displacement and roof displacement as analysis indexes, and each index is evaluated by comparing the results of calculation with or without selfresetting energy dissipation braces.

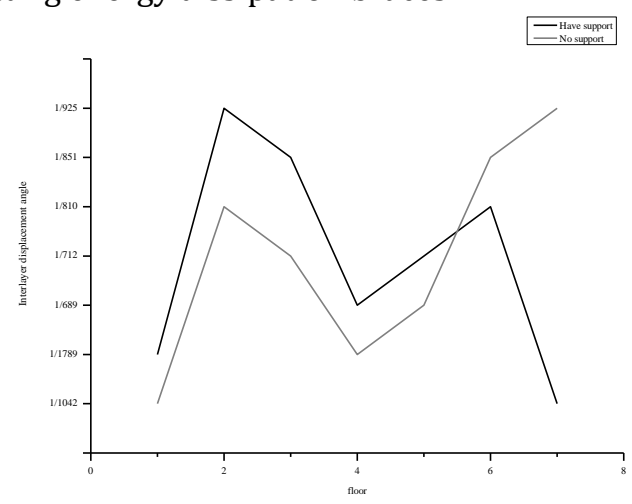

Figure 5: Peak value of interlayer displacement angle under seismic wave action

From the above chart, it can be seen that the peak value of the inter-story displacement angle of some floors of the model structure exceeds the allowable limit when the self-resetting energy dissipation brace is not installed. However, after the selfresetting energy dissipation brace is installed on the fourth and fifth floors of the model structure, since the peak value of the inter-story displacement angle of the two floors is larger, the peak value of the interstory displacement angle of each floor decreases in varying degrees, and meets the allowable limits of the code.

After installing self-resetting energy dissipation braces, the maximum inter-story displacement angles of the model structure in the east-west direction and the north-south direction are reduced by $20.4 \%$ on average under two kinds of frequent earthquake conditions. 


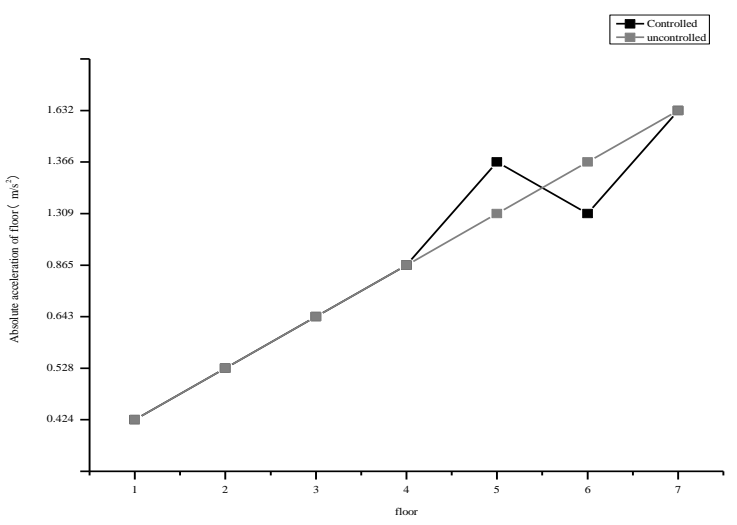

Figure 6: Peak value of absolute acceleration of floor under earthquake wave $\left(\mathrm{m} / \mathrm{s}^{2}\right)$

From the above charts, it can be seen that the absolute acceleration peak response of most floors of the model structure decreases after installing selfreset energy dissipation braces, and the absolute acceleration of the maximum floors in east-west and north-south directions decreases by an average of $34.3 \%$ under two kinds of frequent earthquakes.

This shows that the energy dissipation and seismic strengthening scheme of a building proposed here is reliable.

\section{Conclusion}

The theme is "structure reduction control based on self-reset energy dissipation braces". Firstly, the concrete structure and working principle of selfreset energy dissipation braces are introduced at the level of theoretical research. Then, on the basis of quasi-static test of brace specimens, the finite element modeling and hysteretic performance of braces are numerically analyzed by using general finite element analysis software, and the relevant design parameters are also given. Finally, the threedimensional model of the structure is established in the general finite element analysis software, and the energy dissipation effect of self-reset energy dissipation braces is evaluated by time history analysis. By summarizing the research results, the following conclusions are drawn.

Firstly, steel channel damper has stable hysteretic performance and can consume a lot of energy. Dampers with long or narrow ribs are more flexible, while dampers with shorter or wider ribs have higher stiffness, dissipate energy at a higher rate, but suffer damage relatively early.

Secondly, in the case of reasonable design of seismic reduction and reinforcement scheme, selfresetting energy dissipation brace can give full play to its energy dissipation performance and resetting ability in structure, and achieve better energy dissipation and seismic reduction effect.

After a certain number of self-resetting energy dissipation braces are installed in a building, the seismic performance indexes of the model structure under frequent earthquakes, such as inter-story displacement angle, absolute acceleration of floor and bottom shear force, are reduced in varying degrees.

\section{References}

[1] Konow, N., Roberts, T. J. (2015). The series elastic shock absorber: tendon elasticity modulates energy dissipation by muscle during burst deceleration. Proc Biol Sci, 282(1804), 20142800.

[2] Gaugler, M., Wirz, D., Ronken, S. (2015). Fibrous cartilage of human menisci is less shockabsorbing and energy-dissipating than hyaline cartilage. Knee Surgery Sports Traumatology Arthroscopy, 23(4), 1141-1146.

[3] Singh, S., Satpute, N. V. (2015). Design and analysis of energy-harvesting shock absorber with electromagnetic and fluid damping. Journal of Mechanical Science \& Technology, 29(4), 15911605.

[4] Li, S., Guo, A., Li, H. (2016). An analysis of pounding mitigation and stress waves in highway bridges with shape memory alloy pseudo-rubber shock-absorbing devices. Structural Control \& Health Monitoring, 23(10), 1237-1255.

[5] Pu, Z., Heyne, M. A., To, A. C. (2015). Biomimetic Staggered Composites with Highly Enhanced Energy Dissipation: Design, Modeling, and Test. Journal of the Mechanics \& Physics of Solids, 83, 285-300.

[6] Travanca, J., Hong, H. (2015). Energy dissipation in high-energy ship-offshore jacket platform collisions. Marine Structures, 40, 1-37.

[7] Correagalvis, V., Poschmann, G., Melzer, M. (2016). PsbS interactions involved in the activation of energy dissipation in Arabidopsis. Nat Plants, 2(2), 15225.

[8] Martínez-Hergueta, F., Ridruejo, A., González, C. (2015). Deformation and energy dissipation mechanisms of needle-punched nonwoven fabrics: A multiscale experimental analysis. International Journal of Solids \& Structures, 64$65,120-131$.

[9] Luna Vera, O. S., Kim, C. W., Oshima, Y. (2017). Energy dissipation and absorption capacity influence on experimental modal parameters of a PC girder. Journal of Physics: Conference Series, 842, 012038.

[10] Bashtovoi, V., Motsar, A., Reks, A. (2017). Energy dissipation in a finite volume of magnetic fluid. Journal of Magnetism \& Magnetic Materials, 431, 245-248.

[11] Mohammadzadeh-Habili, J., Heidarpour, M., Samiee, S. (2018). Study of Energy Dissipation and Downstream Flow Regime of Labyrinth Weirs. Iranian Journal of Science \& Technology Transactions of Civil Engineering, 42(2), 1-9. 